\title{
Novobiocin. Redesigning a DNA Gyrase Inhibitor For Selective Inhibition of Hsp90
}

\author{
Joseph A. Burlison, ${ }^{1}$ Len Neckers, ${ }^{2}$ Andy Smith, ${ }^{3}$ Anothony Maxwell, ${ }^{3}$ and \\ Brian S. J. Blagg ${ }^{1 *}$
}

Supporting Information

\footnotetext{
${ }^{1}$ Department of Medicinal Chemistry, The University of Kansas, 1251 Wescoe Hall Drive, Malott Hall 4070, Lawrence, KS 66045-7563.

${ }^{2}$ Urologic Oncology Branch, National Cancer Institute, NIH, Rockville, Maryland 20850.

${ }^{3}$ Department of Biological Chemistry, John Innes Centre, Coney Lane, Norwich NR4 7UH, UK

bblagg@ku.edu
} 
Table of Contents

${ }^{1} \mathrm{H}$ NMR spectrum of 5

${ }^{1} \mathrm{H}$ NMR spectrum of $\mathbf{6}$

${ }^{13} \mathrm{C}$ NMR spectrum of $\mathbf{6}$

${ }^{1} \mathrm{H}$ NMR spectrum of 7

${ }^{13} \mathrm{C}$ NMR spectrum of 7

${ }^{1} \mathrm{H}$ NMR spectrum of $\mathbf{9}$

${ }^{1} \mathrm{H}$ NMR spectrum of $\mathbf{1 3}$

S9

${ }^{1} \mathrm{H}$ NMR spectrum of $\mathbf{1 5}$

S10

${ }^{13} \mathrm{C}$ NMR spectrum of $\mathbf{1 5}$

S11

${ }^{1} \mathrm{H}$ NMR spectrum of $\mathbf{1 6}$

S12

${ }^{13} \mathrm{C}$ NMR spectrum of $\mathbf{1 6}$

S13

${ }^{1} \mathrm{H}$ NMR spectrum of $\mathbf{1 7}$

S14

${ }^{13} \mathrm{C}$ NMR spectrum of $\mathbf{1 7}$

S15

${ }^{1} \mathrm{H}$ NMR spectrum of DHN1

S16

${ }^{13} \mathrm{C}$ NMR spectrum of DHN1

S17

${ }^{1} \mathrm{H}$ NMR spectrum of DHN2

S18

${ }^{13} \mathrm{C}$ NMR spectrum of DHN2

S19 


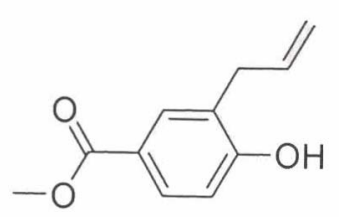

5

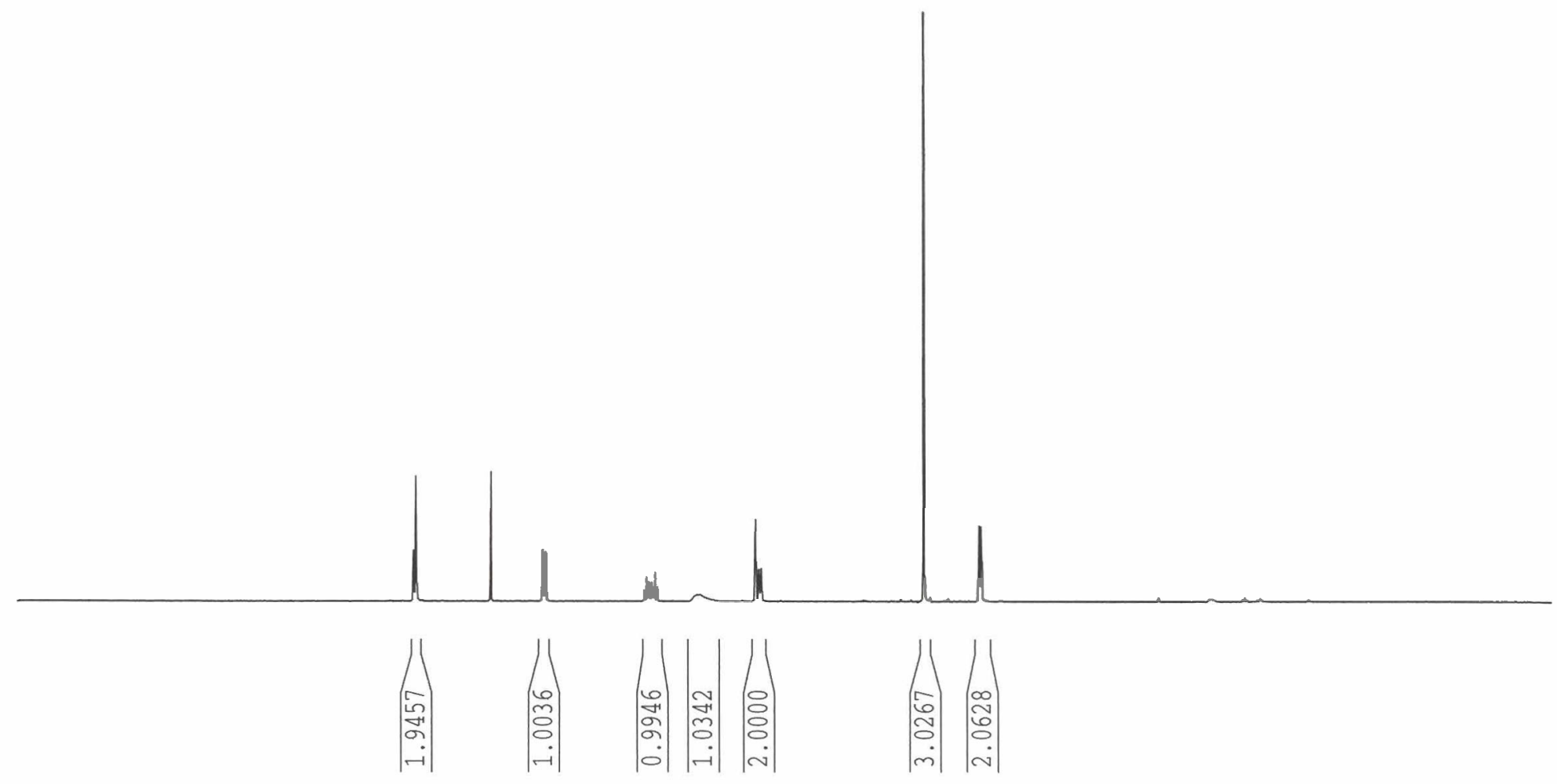

Current Data Parameters

NAME Claisen allyl $11-9$

BXPNO

82 - Acquisition Parameters

Date_ 20051109

Time $\quad 11.34$

INSTROM 400

PROBHD 5 ma ONP 1H/13

PULPROG $\quad$ zg30

$\begin{array}{ll}\text { TD } & 32768 \\ \text { SOLVENT } & \text { CDC13 }\end{array}$

SOLVENI

DS

SWH $\quad 278$

FIDRES $\quad 0.146157 \mathrm{~Hz}$

AO $\quad 3.4210291 \mathrm{sec}$

$\mathrm{RG}$

TE

KCRE

MCWR

362

104.400 usec 5.50 usec $293.2 \mathrm{~K}$

$1.00000000 \mathrm{sec}$ $0.00000000 \mathrm{sec}$ $0.01500000 \mathrm{sec}$

$\begin{array}{lr}==z====\text { CHANNEL } & \text { E1 }======= \\ \text { NUC1 } & 1 \mathrm{H} \\ \text { P1 } & 9.35 \text { usec } \\ \text { PL1 } & -4.00 \mathrm{~dB} \\ \text { SFO1 } & 400.1320007 \mathrm{MHz}\end{array}$

E2 - Processing parameters

SI 32768

SF $\quad 400.1300091$

WDW 400.1300091

SSB $\quad 0$

$\begin{array}{lc}\text { LB } & 0.30 \mathrm{~Hz} \\ \text { GB } & 0\end{array}$

$\begin{array}{lr}\mathrm{GB} & 0 \\ \mathrm{PC} & 1.00\end{array}$

1D MMR plot parameters

CX $\quad 20.00 \mathrm{~cm}$

CY $\quad 0.00 \mathrm{~cm}$

$\begin{array}{ll}\text { F1P } & 10.962 \mathrm{ppm} \\ \text { F1 } & 4386.17 \mathrm{~Hz}\end{array}$

F2P $\quad-1.007 \mathrm{ppm}$

F2 $\quad-403.10 \mathrm{~Hz}$

PPMCM $\quad 0.59846 \mathrm{ppm} / \mathrm{cm}$

HZCM $239.46358 \mathrm{~Hz} / \mathrm{cm}$ 


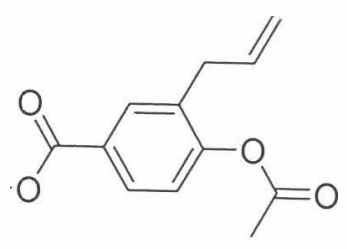

6

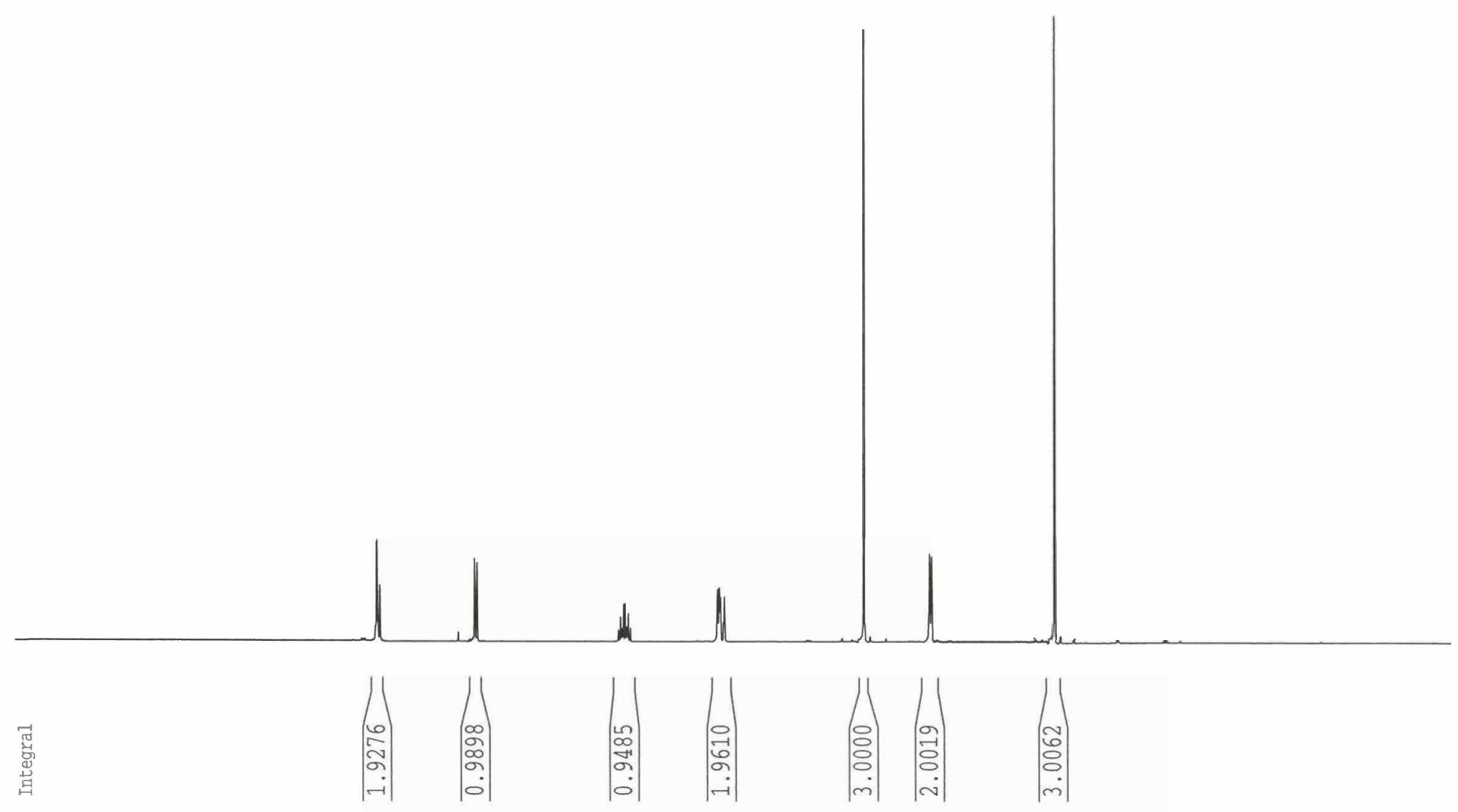

Current Data Parameters

NAME allyl acetate 11-28

EXPNO

PROCNO

F2 - Acquisition Parameters

Date_ 20051128

Time $\quad 13.46$

INSTRUM $\quad \operatorname{drx} 400$

PROBHD $5 \mathrm{~mm}$ QNP $1 \mathrm{H} / 13$

PULPROG 293

$\begin{array}{ll}\text { TD } & 32768 \\ \text { SOLVENT } & \text { CDCl3 }\end{array}$

NS
DS

$\begin{array}{lr}\text { DS } & 2 \\ \text { SWH } & 4789.272 \\ \text { FIDRES } & 0.146157\end{array}$

AQ $\quad 3.4210291 \mathrm{sec}$

$\begin{array}{lr}\text { RG } & 57 \\ D W & 104.400\end{array}$

$\begin{array}{lr}\text { DW } & 104.400 \text { usec } \\ D E & 5.50 \text { usec }\end{array}$

$\begin{array}{lr}\mathrm{DE} & 5.50 \mathrm{us} \\ \mathrm{TE} & 293.2 \mathrm{R}\end{array}$

D1 $\quad 1.00000000 \mathrm{sec}$

MCREST $\quad 0.00000000 \mathrm{sec}$

$\begin{array}{ll}\text { MCWRK } & 0.01500000 \mathrm{sec}\end{array}$

$=======$ CHANNEL $\mathrm{f1}= \pm===$

NUC1

9.35 usec

SF01 $400-\frac{-4.00 \mathrm{~dB}}{1320007}$

F2 - Processing parameters

SI

SF $\quad 400.1300091 \mathrm{MHz}$

WDW $\quad$ EM

$\begin{array}{ll}\text { SSB } & 0 \\ \text { LB } & 0.30\end{array}$

$\mathrm{LB}$
$\mathrm{GB}$
$\mathrm{PC}$

$0.30 \mathrm{~Hz}$
$\mathrm{O}$

1.00

ID NMR plot parameters

CX $\quad 20.00 \mathrm{~cm}$

$\begin{array}{ll}C Y & 0.00 \mathrm{~cm}\end{array}$

F1P $\quad 10.962 \mathrm{ppm}$

$\begin{array}{lr}\text { F1 } & 4386.17 \mathrm{~Hz} \\ \text { F2P } & -1.007 \mathrm{gpm}\end{array}$

F2 $\quad-403.10 \mathrm{~Hz}$

PPMCM $\quad 0.59846 \mathrm{ppm} / \mathrm{c}$

$$
239.46358 \mathrm{~Hz} / \mathrm{CII}
$$




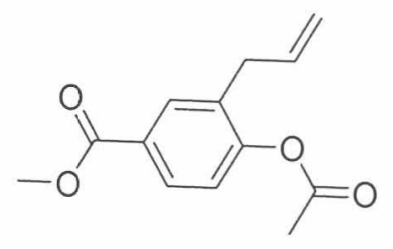

6

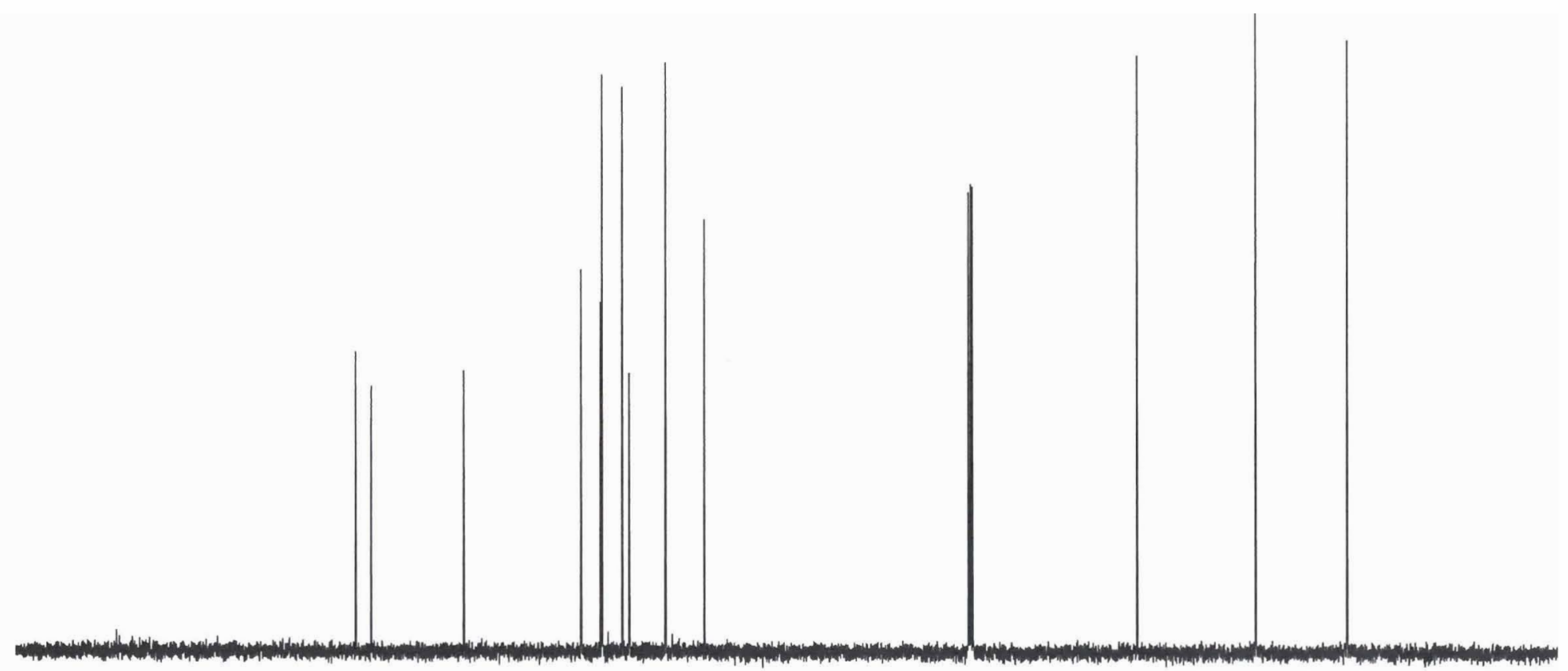

pringt $200 ; 175$

150
75
Current Data Parameters

NAME allyl acetate 11-2

EXPNO

2
1

F2 - Acquisition Parameters

Date_ $\quad 20051128$

Time $\quad 13.51$

INSTRUM drx400

PROBHD $5 \mathrm{Mm}$ QNP $1 \mathrm{H} / 13$

PULPROG 2gpg30

TD $\quad 65536$

$\begin{array}{lr}\text { SOLVENT } & \text { CDC13 } \\ \text { NS } & 200\end{array}$

SWH $\quad 23148.148 \mathrm{~Hz}$

$0.353213 \mathrm{~Hz}$

$\mathrm{AQ} \quad 1.4156276 \mathrm{sec}$

$\begin{array}{ll}\text { RG } & 2896.3 \\ \text { DW } & 21.600 \text { usec }\end{array}$

$\begin{array}{lr}\text { DW } & 21.600 \text { usec } \\ D E & 5.50 \text { usec }\end{array}$

$\mathrm{TE}-294.2 \mathrm{~K}$

D1 $\quad 0.15000001 \mathrm{sec}$

di1 $\quad 0.05000000 \mathrm{sec}$

DELTA $\quad 0.05000000 \mathrm{sec}$

$0.0150000 \mathrm{sec}$

(1) =

$== \pm=====$ CHANNEL f1 $=======$
NUC1 $13 \mathrm{C}$

$\begin{array}{lr}\text { NUC1 } & 13 \mathrm{C} \\ \text { P1 } & 11.25 \text { usec }\end{array}$

$2.00 \mathrm{~dB}$

$100.6232933 \mathrm{MHz}$

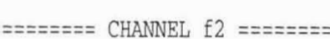

CPDPRG2 waltz16

$\begin{array}{lc}\text { NUC2 } & 1 \mathrm{H} \\ \text { PCPD2 } & 100.00 \mathrm{usec}\end{array}$

PL2 $\quad-4.00 \mathrm{~dB}$

$16.58 \mathrm{~dB}$

$\begin{array}{lr}\text { PL13 } & 17.00 \mathrm{~dB} \\ \text { SF02 } & 400.1316005 \mathrm{MHz}\end{array}$

F2 - Processing parameters

$\begin{array}{lc}\text { F2 } & \text { - Processing parameters } \\ \text { SI } & 65536 \\ \text { SF } & 100.6127290 \mathrm{MHz}\end{array}$

$\begin{array}{lr}\text { SF } & 100.6127290 \\ \text { WDW } & \text { EM }\end{array}$

$\begin{array}{lr}\text { SSB } & 0 \\ \mathrm{LB} & 1.00 \mathrm{~Hz}\end{array}$

$\begin{array}{lr}\text { GB } & 0 \\ \text { PC } & 1.40\end{array}$

1D NMR plot parameters

CX $\quad 20.00 \mathrm{~cm}$

$\begin{array}{ll}\text { CY } & 0.00 \mathrm{~cm}\end{array}$

F1 $220.036 \mathrm{ppm}$

$\begin{array}{lr}\text { F1 } & 22138.42 \mathrm{~Hz} \\ \text { F2P } & -10.036 \mathrm{ppm}\end{array}$

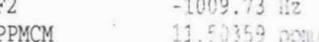

PPMCM $\quad 11.50350$ 


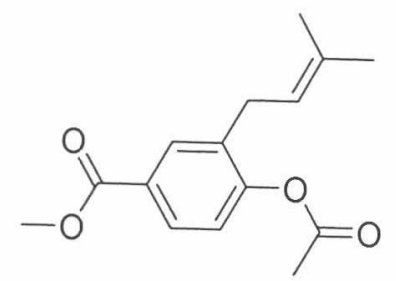

7

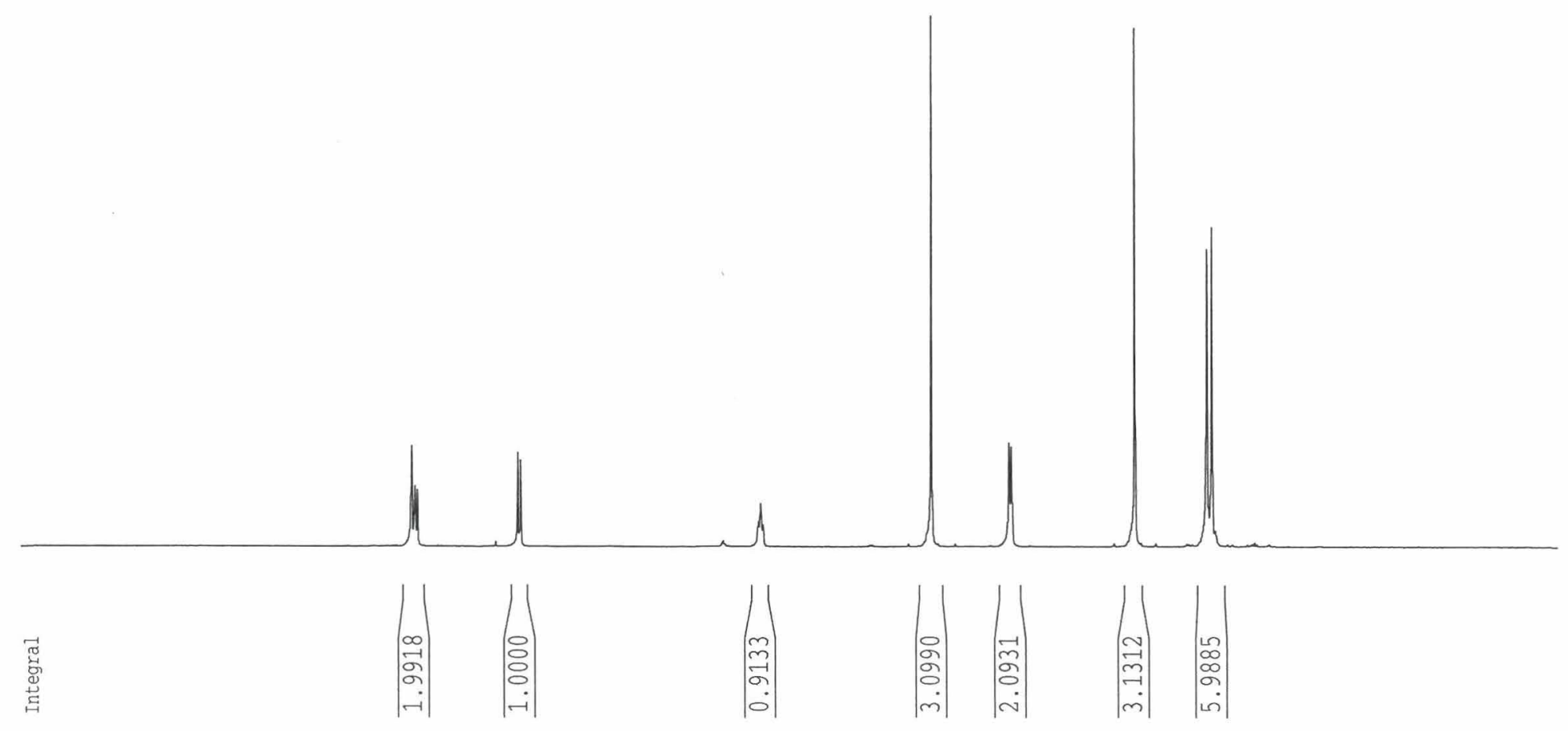

Current Data Parameters

NAMR prenyl acetate 11-29

PROCNO

R2 - Acquisition Parameters Date_ 20051129

Time $\quad 12.14$ INSTRUM drx400 PROBHD $5 \mathrm{~mm}$ QNP $1 \mathrm{H} / 13$ PULPROG $\quad$ zg30 TD 32768 SOLVEWT

NS $\begin{array}{ll}\text { DS } & 2 \\ \text { SWH } & 4789.272 \mathrm{~Hz}\end{array}$ FIDRES $\quad 0.146157 \mathrm{~Hz}$ AQ $\quad 3.4210291 \mathrm{sec}$ RG $\quad 35.9$ DW $\quad 104.400$ usec $\begin{array}{ll}\mathrm{DE} & 5.50 \mathrm{usec} \\ \mathrm{TE} & 293.2 \mathrm{k}\end{array}$ D1 $\quad 1.00000000 \mathrm{sec}$ MCREST $\quad 0.00000000 \mathrm{sec}$ MCWRK $\quad 0.01500000 \mathrm{sec}$

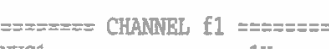
$\begin{array}{lc}\text { NUC1 } & 1 \mathrm{H} \\ \text { P1 } & 9.35 \text { usec }\end{array}$ P1 1 SFO1 $\quad 400.1320007 \mathrm{kHz}$

F2 - Processing parameters SI 32768 SF $\quad 400.1300091 \mathrm{MHz}$ WDW $\begin{array}{ll}\text { SSB } & 0 \\ \text { LB } & 0.30 \mathrm{~Hz}\end{array}$ $\begin{array}{lr}\mathrm{GB} & 0 \\ \mathrm{PC} & 1.00\end{array}$

1D NMR plot parameters CX $20.00 \mathrm{cma}$ $\begin{array}{lc}\text { CY } & 0.00 \mathrm{~cm} \\ \text { F1P } & 10.962 \mathrm{ppm}\end{array}$ $\begin{array}{ll}\text { F1 } & 4386.17 \mathrm{~Hz} \\ \text { F2p } & -1.007 \mathrm{ppm}\end{array}$ $\begin{array}{ll}\text { F2P } & -1.007 \mathrm{ppm} \\ \text { F2 } & -403.10 \mathrm{~Hz}\end{array}$ PPYCM $\quad 0.59846 \mathrm{ppm} / \mathrm{cm}$ Hitar $239.46358 \mathrm{~Hz} / \mathrm{cm}$ 


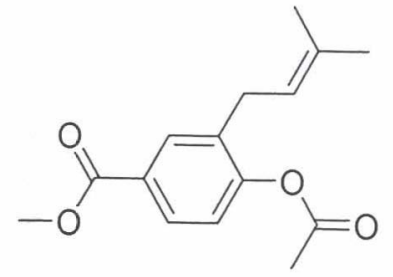

7
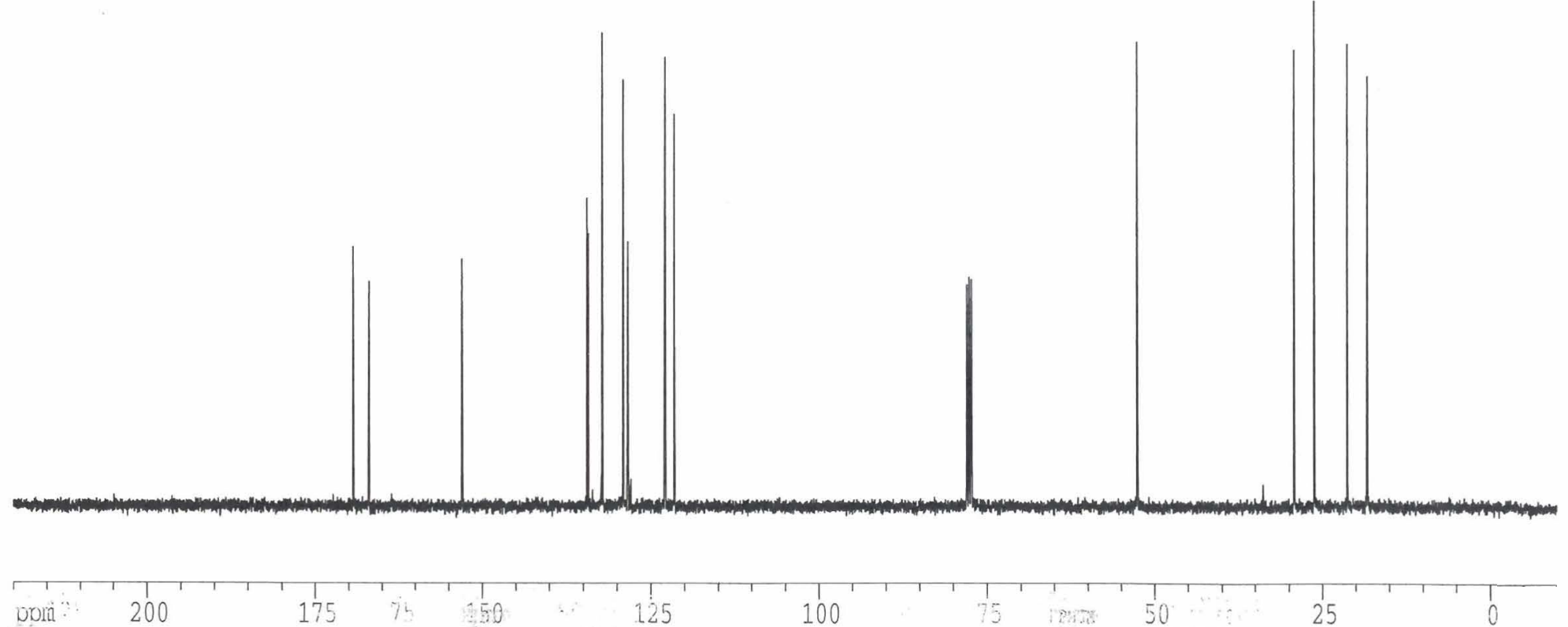

200

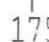

$175 \quad 7$

150

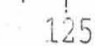

100
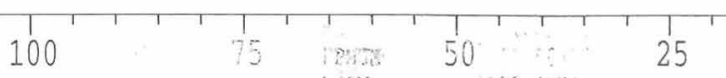

S7
Current Data Parameters

NAME prenyl acetate 11-29

PROCNO

2
1

F2 - Acquisition Parameters

Date_ 20051129

Time $\quad 12.20$

INSTRUM drx400

PROBHD $5 \mathrm{~mm}$ QNP $1 \mathrm{H} / 13$

$\begin{array}{lr}\text { PULPROG } & \text { zgpg30 } \\ \text { TD } & 65536\end{array}$

SOLVENT CDC13

NS

DS

SWH

$23148.148 \mathrm{~Hz}$

FIDRES $\quad 0.353213 \mathrm{~Hz}$

AQ $\quad 1.4156276 \mathrm{sec}$

RG 3251

$\begin{array}{lr}D W & 21.600 \text { usec } \\ D E & 5.50 \text { usec }\end{array}$

TE $293.2 \mathrm{~K}$

D1 $\quad 0.15000001 \mathrm{sec}$

d11 $\quad 0.03000000 \mathrm{sec}$

DELTA $\quad 0.05000000 \mathrm{sec}$

MCREST $\quad 0.00000000 \mathrm{sec}$

MCWRK $\quad 0.01500000 \mathrm{sec}$

$=== \pm=$ CHANNEL f1 $=======$

NoC1 $13 \mathrm{C}$

$\begin{array}{lr}\text { P1 } & 11.25 \text { usec } \\ \text { PL1 } & 2.00 \mathrm{~dB}\end{array}$

$\begin{array}{lr}\text { PL1 } & 2.00 \mathrm{~dB} \\ \text { SEO1 } & 100.6232933 \mathrm{MHz}\end{array}$

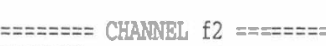

CPDPRG2 waltz216

NUC2 $1 \mathrm{H}$

PCPD2 $\quad 100.00$ usec

$\begin{array}{ll}\text { PL2 } & -4.00 \mathrm{~dB} \\ \text { PL12 } & 16.58 \mathrm{~dB}\end{array}$

$\begin{array}{ll}\text { PL12 } & 16.58 \mathrm{~dB} \\ \text { PL13 } & 17.00 \mathrm{~dB}\end{array}$

$\begin{array}{lr}\text { PL13 } & 17.00 \mathrm{~dB} \\ \text { SFO2 } & 400 \quad 1316005 \mathrm{MHz}\end{array}$

F2 - Processing parameters

SI $\quad 65536$

SF $\quad 1006127290 \mathrm{MH}$

WDW

SSB

LB
GB

䁩
0

$1.00 \mathrm{~Hz}$

$1.00 \mathrm{~Hz}$

1.40

1D NMR plot parameters

CX $\quad 20.00 \mathrm{~cm}$

$\begin{array}{lr}\text { CY } & 0.00 \mathrm{~cm} \\ \text { F1P } & 220.036 \mathrm{ppm}\end{array}$

$\begin{array}{lr}\text { F1P } & 220.036 \mathrm{pp} \\ \text { F1 } & 22138.42 \mathrm{~Hz}\end{array}$

F2P $\quad-10.036 \mathrm{ppm}$

F2 $\quad-1009.73 \mathrm{~Hz}$

Pemat $\quad 11.50359 \mathrm{ppm} / \mathrm{cm}$ 


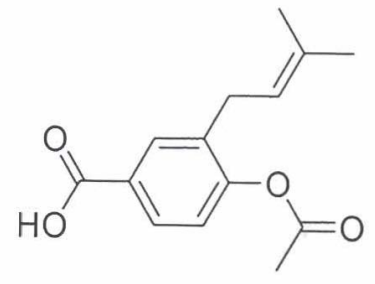

9

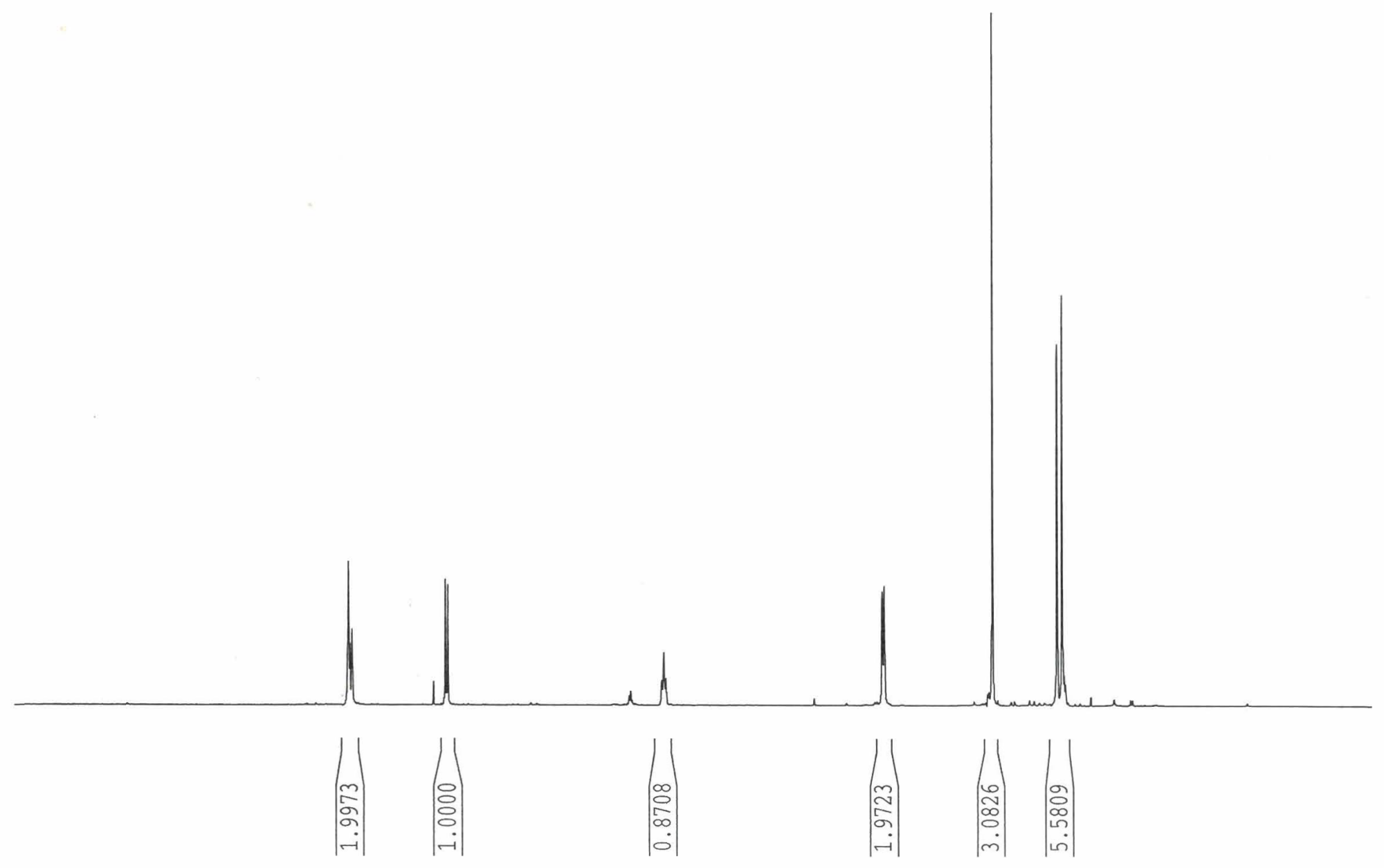

rrent Data Parameters

WE acetate acid 12-5

PNO

oCNO

- Acquisition Parameters

te_ 20051205

$\begin{array}{lr}\text { me } & 13.52\end{array}$

ISTRUM drx 400

DBBDD 5 Intin ONP 1H/13

Z JPROG
32768

$\begin{array}{ll}\text { TD } & 32768 \\ \text { SOLVENT } & \mathrm{CDC13}\end{array}$

solvint

DS

SWII

$4789.272 \mathrm{~Hz}$

$0.146157 \mathrm{kz}$

RG

DE

TQ

D1

MCWRK

II

NUC1

PI

SFOI

$1 \mathrm{H}$
$.35 \mathrm{usec}$

$-4.00 \mathrm{~dB}$

$400.1320007 \mathrm{MHz}$

72 - Processing parameters

$\begin{array}{lc}\text { SI } & 32768 \\ \text { SP } & 400.1300091 \mathrm{MHz}\end{array}$

WDW

SSB

$\mathrm{LB}$
$\mathrm{GB}$

BC

D NMR plot parameters

C\& $20.00 \mathrm{~cm}$

CY $\quad 0.00 \mathrm{~cm}$

F1P 10.962 ppm

F1 $4386.17 \mathrm{Bz}$

$\begin{array}{ll}\text { F2P } & -1.007 \mathrm{ppm} \\ \text { F2 } & -403.10 \mathrm{~Hz}\end{array}$

PPMCM $\quad 0.59846 \mathrm{ppm} / \mathrm{cm}$

HZCM $\quad 239.46358 \mathrm{~Hz} / \mathrm{cm}$ 


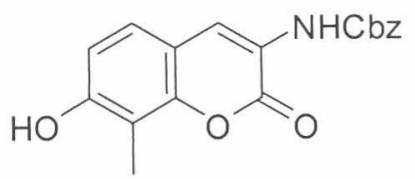

13

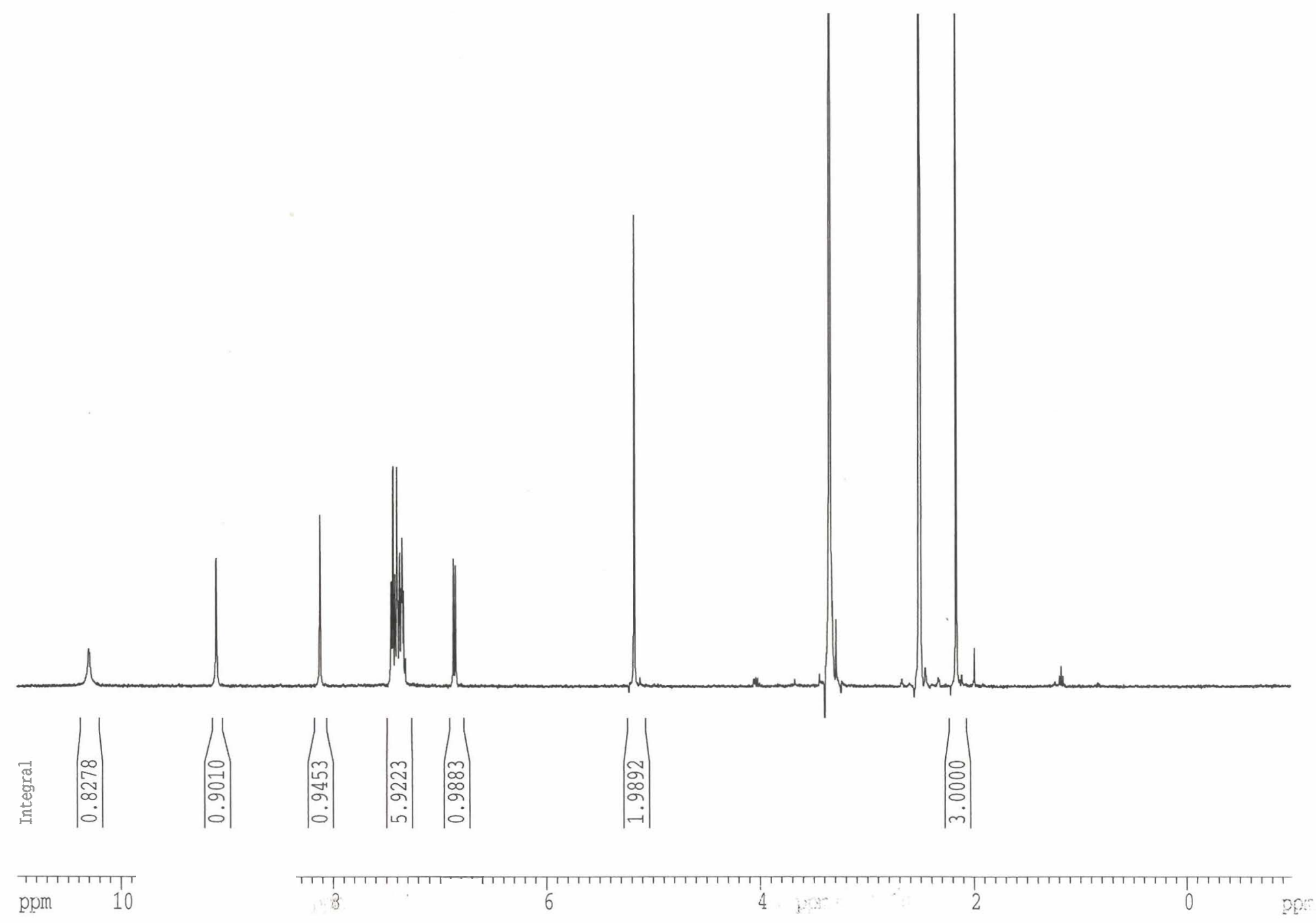

Current Data Parameters NAME

PROCNO

F2 - Acquisition Parameters

Date_ 20050906

$\begin{array}{lr}\text { Time } & 8.14\end{array}$

PROBHD $5 \mathrm{~mm}$ NP 1 $\mathrm{z} / 13$

PULPROG $\quad$ gg 30

TD 32768

SOLVENTT DYSO

NS

SWH $\quad 4789.272 \mathrm{~Hz}$

FIDRES $\quad 0.146157 \mathrm{~Hz}$

AQ $\quad 3.4210291 \mathrm{sec}$

RG 512

DW $\quad 104.400$ usec

DE $\quad 5.50$ usec

DE
TE

D1 $\quad 1.00000000 \mathrm{sec}$

MCREST $\quad 0.00000000 \mathrm{sec}$

MCWRK $\quad 0.01500000 \mathrm{sec}$

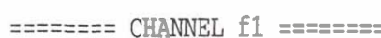
NJC1 1

P1 9.35 useec

PL1 $\quad-4.00 \mathrm{~dB}$

SFO1 $400.1320007 \mathrm{MH}$

F2 - Processing parameters

SI 32768

SF $\quad 400.1300000 \mathrm{MHz}$

WDW EN

$\begin{array}{lc}\text { SSB } & 0 \\ \text { LB } & 0.30 \mathrm{~Hz}\end{array}$

$\begin{array}{lr}\text { GB } & 0 \\ \text { PC } & 1.00\end{array}$

1D MMR plot paraneters

$\begin{array}{lr}\text { CX } & 20.00 \mathrm{~cm} \\ \text { CY } & 0.00 \mathrm{~cm}\end{array}$

F1P $\quad 10.985 \mathrm{ppm}$

$4395.28 \mathrm{~Hz}$

-0.985 ppm

$\begin{array}{ll}\text { F2 } & -393.99 \mathrm{~Hz} \\ \text { PPNC } & 0.59846 \mathrm{ppm} / \mathrm{cm}\end{array}$

$\begin{array}{ll}\text { F2 } & -393.99 \mathrm{~Hz} \\ \text { PPNC } & 0.59846 \mathrm{ppm} / \mathrm{cm}\end{array}$

HZCM $\quad 239.46362 \mathrm{~Hz} / \mathrm{cm}$

\author{
(3.46362 $\mathrm{Hz} / \mathrm{cm}$
}




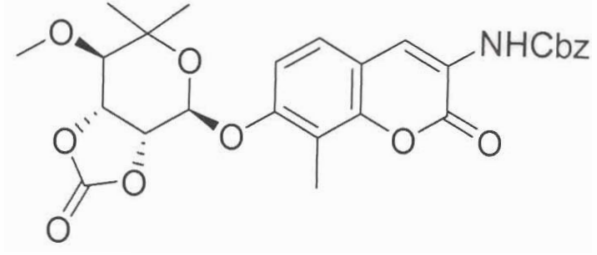

15

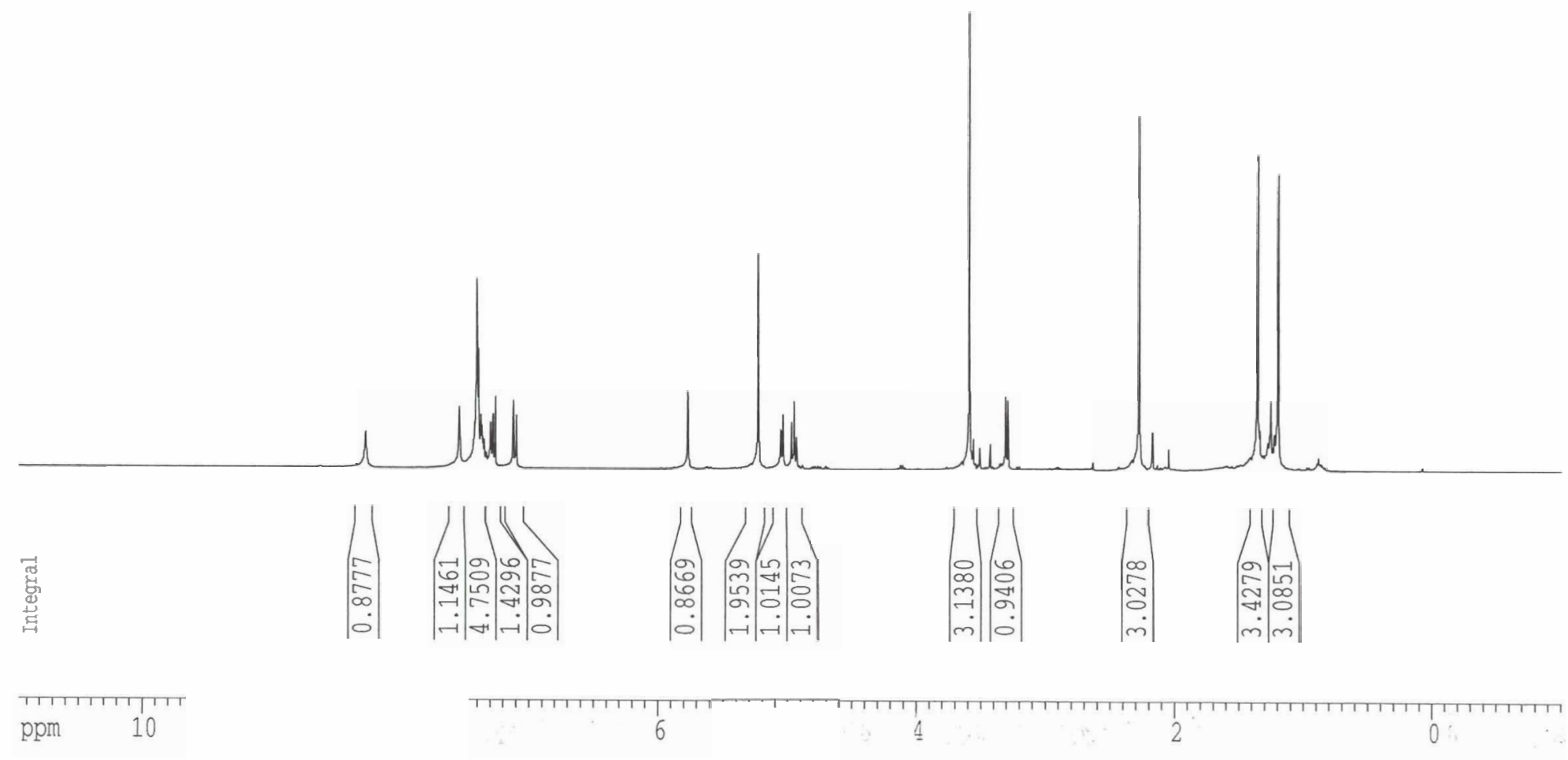

Current Data Parameters CBZ 1-5-06

EXPNO

PROCNO

F2 - Acquisition Parameters

Date_ 20060105

Time $\quad 8.06$

INSTRUM drX400

PROBHD $5 \mathrm{~mm}$ QNP 1H/13

PULPROG $\quad \mathrm{zg} 30$

$\begin{array}{ll}\text { TD } & 32768 \\ \text { SOLVENT } & \end{array}$

NS

DS

$\begin{array}{ll}\text { SWH } & 4789.272 \mathrm{~Hz} \\ \text { FIDRES } & 0.146157 \mathrm{~Hz}\end{array}$

$\begin{array}{lc}\text { FIDRES } & 0.146157 \mathrm{~Hz} \\ \mathrm{AQ} & 3.4210291 \mathrm{sec}\end{array}$

RG 143.7

DW $\quad 104.400$ usec

DE $\quad 5.50$ usec

D1 293.2 K

MCREST $\quad 0.00000000 \mathrm{sec}$

MCREST $0.00000000 \mathrm{sec}$ MCWRK $\quad 0.01500000 \mathrm{sec}$

$===== \pm==$ CHANNELL $\mathrm{f} 1$ === ==== NUC1 $1 \mathrm{H}$

P1 9.35 usec

$00 \mathrm{~dB}$

$4001320007 \mathrm{MHz}$

F2 - Processing parameters

SI 32768

SF $\quad 400.1300091 \mathrm{MHz}$

WSB EM

LB $\quad 0.30 \mathrm{~Hz}$

GB

PC

0
.00

1D NMR plot parameters

CX $\quad 20.00 \mathrm{~cm}$

$\mathrm{CY} \quad 0.00 \mathrm{~cm}$

F1P $\quad 10.962 \mathrm{ppm}$

$10.962 \mathrm{pp}$

$-1.007 \mathrm{ppm}$

$-1.007 \mathrm{ppm}$
$-403.10 \mathrm{~Hz}$

$\begin{array}{lr}\text { F2 } & -403.10 \mathrm{~Hz} \\ \text { PPMCM } & 0.59846 \mathrm{ppm} / \mathrm{cm} \\ \text { HZCM } & 239.46359 \mathrm{~Hz} / \mathrm{cm}\end{array}$ 


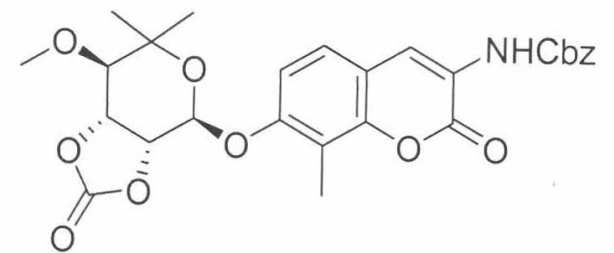

15

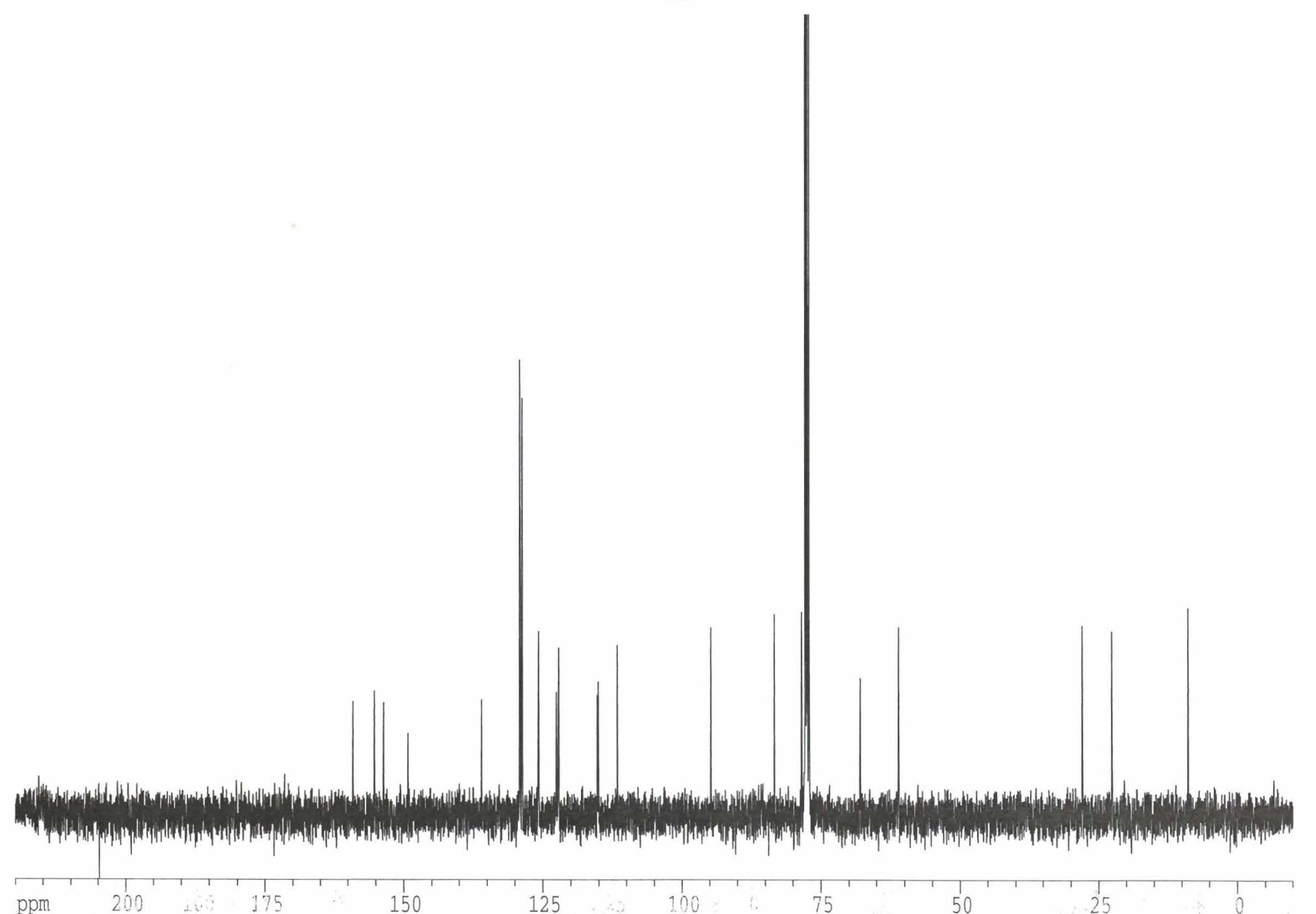

rent Data Parameters

E CBZ 1-5-06

CNO

- Acquisitín Parameter

e_ 20060105

ie

ITRUM drख 400

IBHD 5 man QNAP 1B/13

$\begin{array}{lr}\text { YULPROG } & \text { 29pg30 } \\ \text { TD } & 65536\end{array}$

SOLVENT CDCI3

NS

DS

FIDPFS $\quad 23148.148 \mathrm{~Hz}$

AQ $\quad 0.353213 \mathrm{~Hz}$

RG $\quad 4597.6$

DW $\quad 21.600$ usec

DE $\quad 5.50$ usec

D1 $\quad 0.15000001 \mathrm{sec}$

d11 $\quad 0.03000000 \mathrm{sec}$

DELTA $\quad 0.05000000 \mathrm{sec}$

$0.00000000 \mathrm{sec}$

$=======$ CHAMNEL $[1$ ======="

$\begin{array}{lr}\text { NUC1 } & 13 \mathrm{C} \\ \mathrm{P1} & 11.25 \text { usec }\end{array}$

PL1 $\quad 2.00 \mathrm{~dB}$

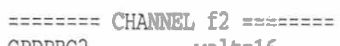

CPDPRG2 waltz16

$\begin{array}{ll}\text { NUC2 } & 1 \mathrm{H} \\ \text { PCPD2 } & 100.00 \text { usec }\end{array}$

PL2 $-4.00 \mathrm{~dB}$

PL12 $16.58 \mathrm{~dB}$

PL13 $\quad 17.00 \mathrm{~dB}$

SF02 $400.1316005 \mathrm{MHz}$

F2 - Processing parameters

SI $\quad 100.6127290 \mathrm{MHz}$

WDW

SSB

$\mathrm{LB}$
$\mathrm{GB}$

$\mathrm{PC}$

1D NMR plot parameters

CX $\quad 20.00 \mathrm{~cm}$

$\begin{array}{ll}\text { F1P } & 0.00 \mathrm{~cm} \\ \mathrm{~F} & 220.036 \mathrm{ppm}\end{array}$

$\begin{array}{lr}\text { F1P } & 220.036 \mathrm{ppm} \\ \text { F1 } & 22138.41 \mathrm{~Hz}\end{array}$

F2P $\quad-10.036 \mathrm{ppm}$

$\begin{array}{ll}\text { P2 } & -1009.74 \mathrm{~Hz} \\ \text { PPMCM } & 11.50359 \mathrm{ppm} / \mathrm{c}\end{array}$

$\quad 1157.50359 \mathrm{ppm} / \mathrm{Cm}$ 


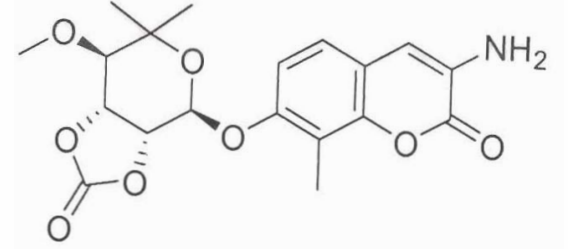

16

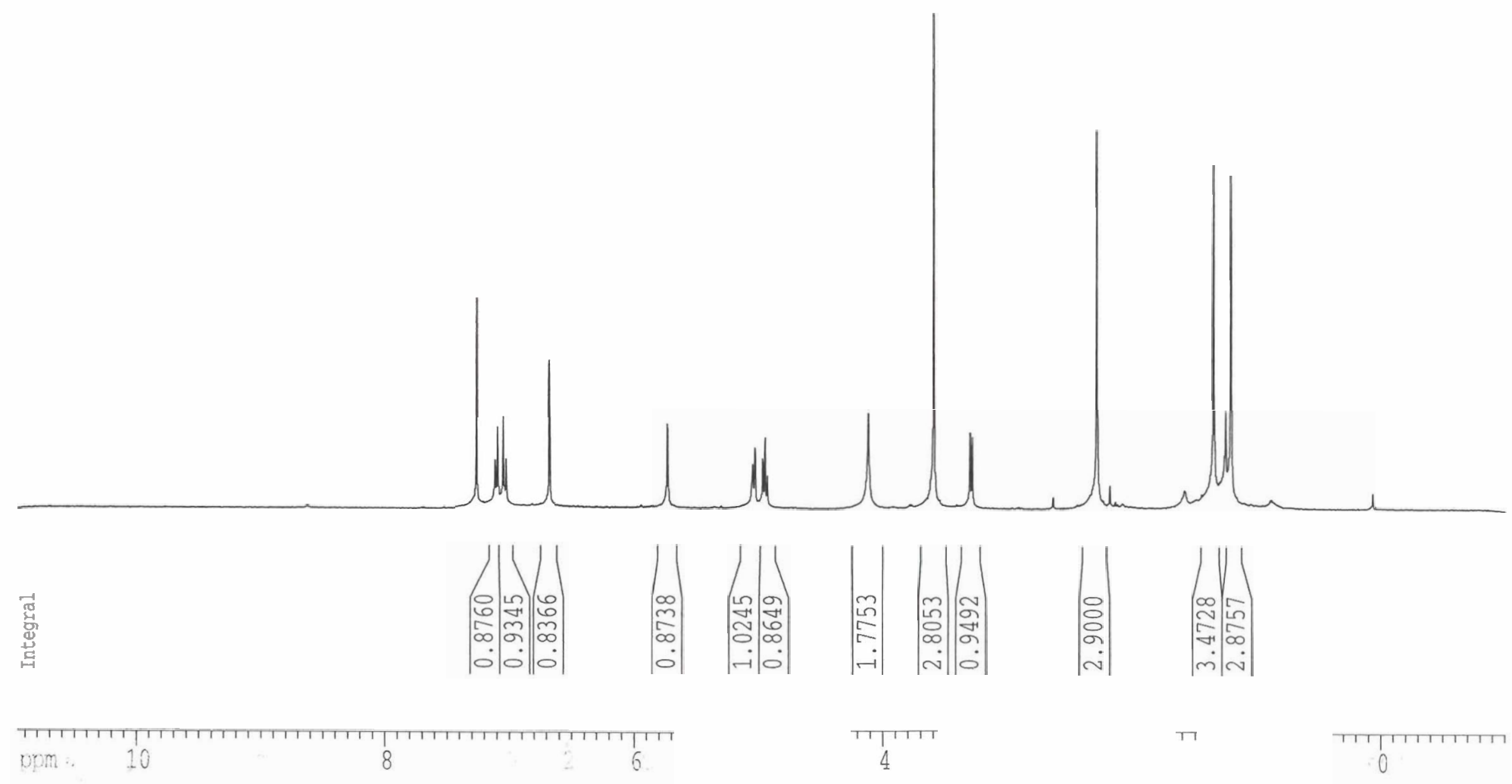

ent Data Parameters NH2 1-5-06

NO

Acquisition Parameters $\begin{array}{lr}e^{2} & 20060105 \\ \text { e } & 7.56\end{array}$ TRUM drx 400 BHD $5 \mathrm{~mm}$ QNP $1 \mathrm{H} / 13$ PROG $\quad \mathrm{gg} 30$ $\begin{array}{ll}\text { TD } & 32768 \\ \text { SOLVENT } & \text { CDC13 }\end{array}$

SWH $\quad 4789.272 \mathrm{~Hz}$ FIDRES $\quad 0.146157 \mathrm{~Hz}$ $\mathrm{AQ} \quad 3.4210291 \mathrm{sec}$ RG 287.4

DW $\quad 104.400$ usec DE $\quad 5.50$ usec $293.2 \mathrm{~K}$ D1 $\quad 1.00000000 \mathrm{sec}$ MCREST $\quad 0.00000000 \mathrm{sec}$ MCWRK $\quad 0.01500000 \mathrm{sec}$

$===-=z==$ CHANNEL f1 $========$ NUC1 $1 \mathrm{H}$

9.35 usec

PL1 $-4.00 \mathrm{~dB}$

SF01 $\quad 400.1320007 \mathrm{MHz}$

F2 - Processing parameters

SI $\quad 32768$

SF $\quad 400.1300091 \mathrm{MHz}$

WDW

LB $\quad 0.30 \mathrm{~Hz}$

GB
PC

$0.30 \mathrm{~Hz}$
$\mathrm{O}$

1.00

1D NMR plot parameters

CX $\quad 20.00 \mathrm{~cm}$

CY $\quad 0.00 \mathrm{cin}$

F1P $\quad 10.962 \mathrm{ppm}$ $4386.17 \mathrm{~Hz}$ $-1.007 \mathrm{ppm}$ $\begin{aligned}-1.007 \mathrm{ppm} & -403.10 \mathrm{~Hz}\end{aligned}$ $-403.10 \mathrm{~Hz}$
$0.59846 \mathrm{ppm} / \mathrm{cm}$ $239.46359 \mathrm{~Hz} / \mathrm{cm}$ 


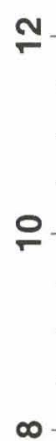

$\infty$

c

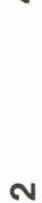

N 0

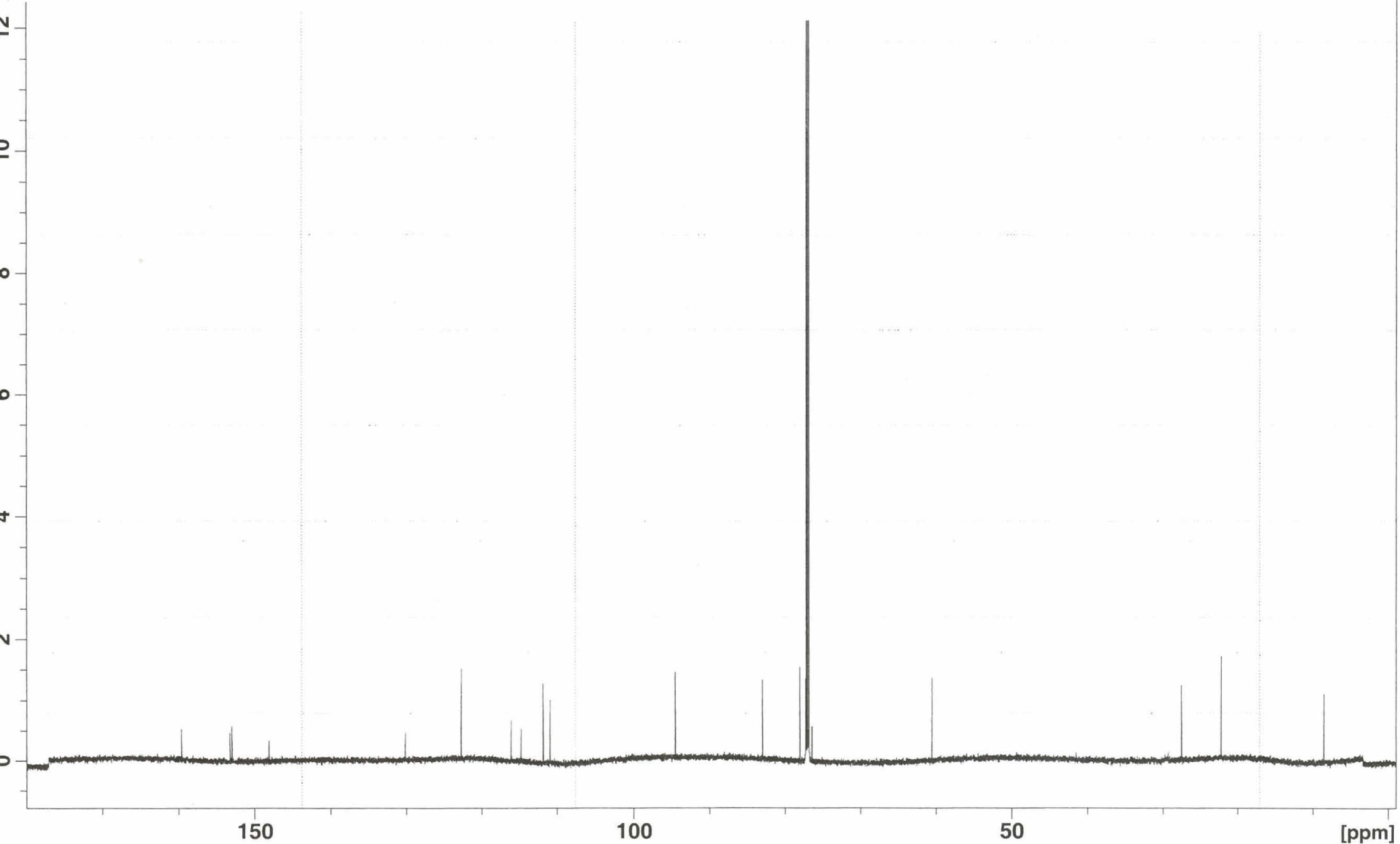




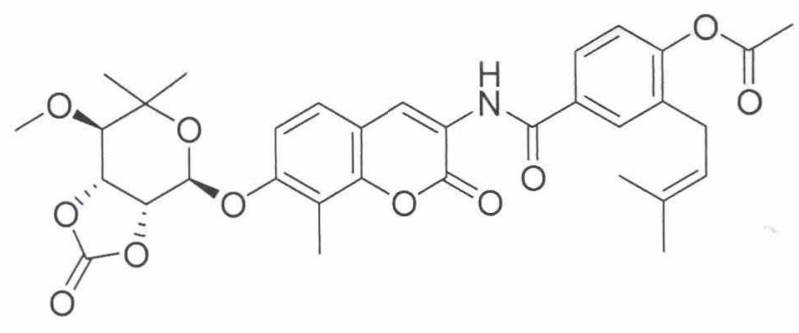

17

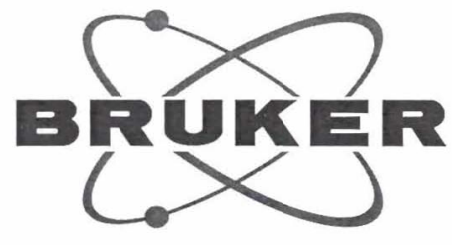

Current Data Parameters NAME

EXPNO

PROCNO

F2 - Acquisition Parameters

Date_ 20051220

Time $\quad 15.42$

INSTRUM

PROBHD

PULPROG

TD

SOLVENT

NS

DS

SWH

FIDRES

$\mathrm{AQ}$

RG

DW

DE

TE

D1

TDO

spect
$\mathrm{mm}$ CPDUL $13 \mathrm{C}$

$\mathrm{zg} 30$

65536

$\mathrm{DCl}$

16
2

2
$10330.578 \mathrm{~Hz}$

$0.157632 \mathrm{~Hz}$

$3.1720407 \mathrm{sec}$$$
\begin{array}{r}
57 \\
57
\end{array}
$$

48.400 usec 6.00 usec $298.0 \mathrm{~K}$

$1.00000000 \mathrm{sec}$

$==x=====$ CHANNEL f1 $==x==x==$ NUC1

P1

PL1

4. 90 usec $2.20 \mathrm{~dB}$

$500.1330885 \mathrm{MHz}$

F2 - Processing parameters

SI 32768

SF $\quad 500.1300466 \mathrm{MHz}$

WDW

SSB

LB

GB

$\mathrm{PC}$

\section{$\begin{array}{llllllllllllllllllll}9.5 & 9.0 & 8.5 & 8.0 & 7.5 & 7.0 & 6.5 & 6.0 & 5.5 & 5.0 & 4.5 & 4.0 & 3.5 & 3.0 & 2.5 & 2.0 & 1.5 & 1.0 & 0.5 & \mathrm{ppm}\end{array}$}

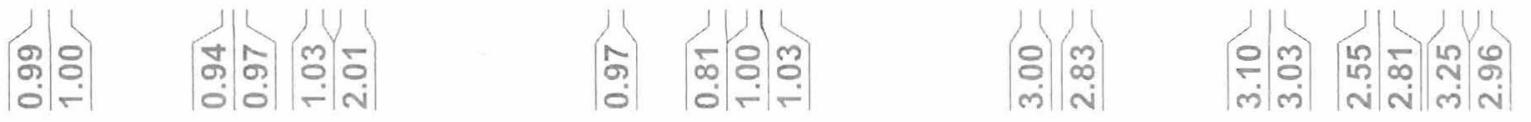




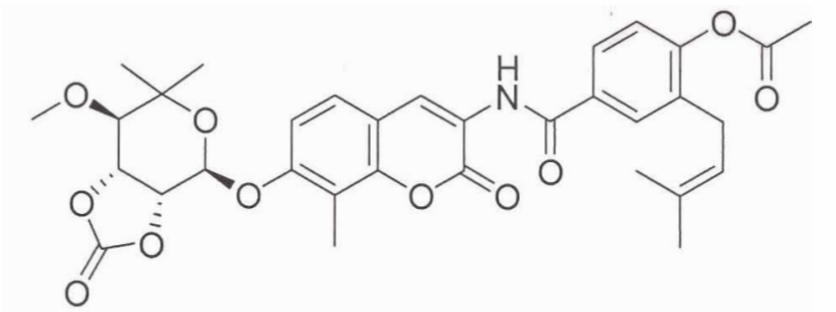

17

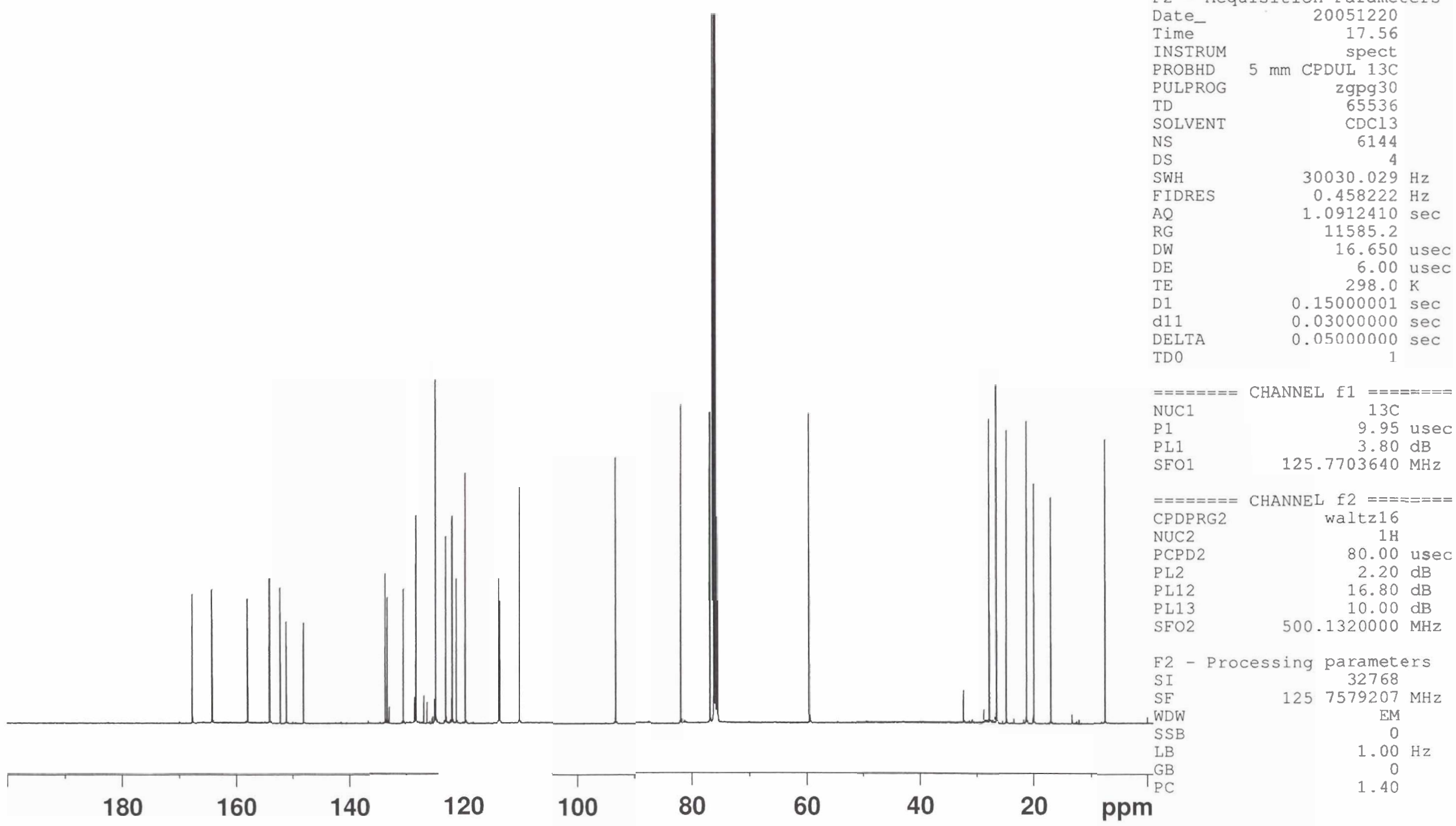




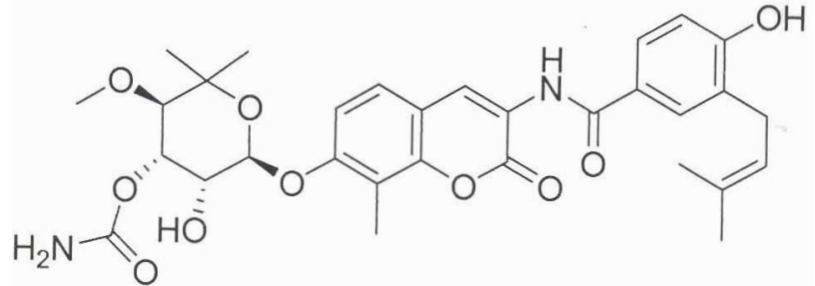

DHN1

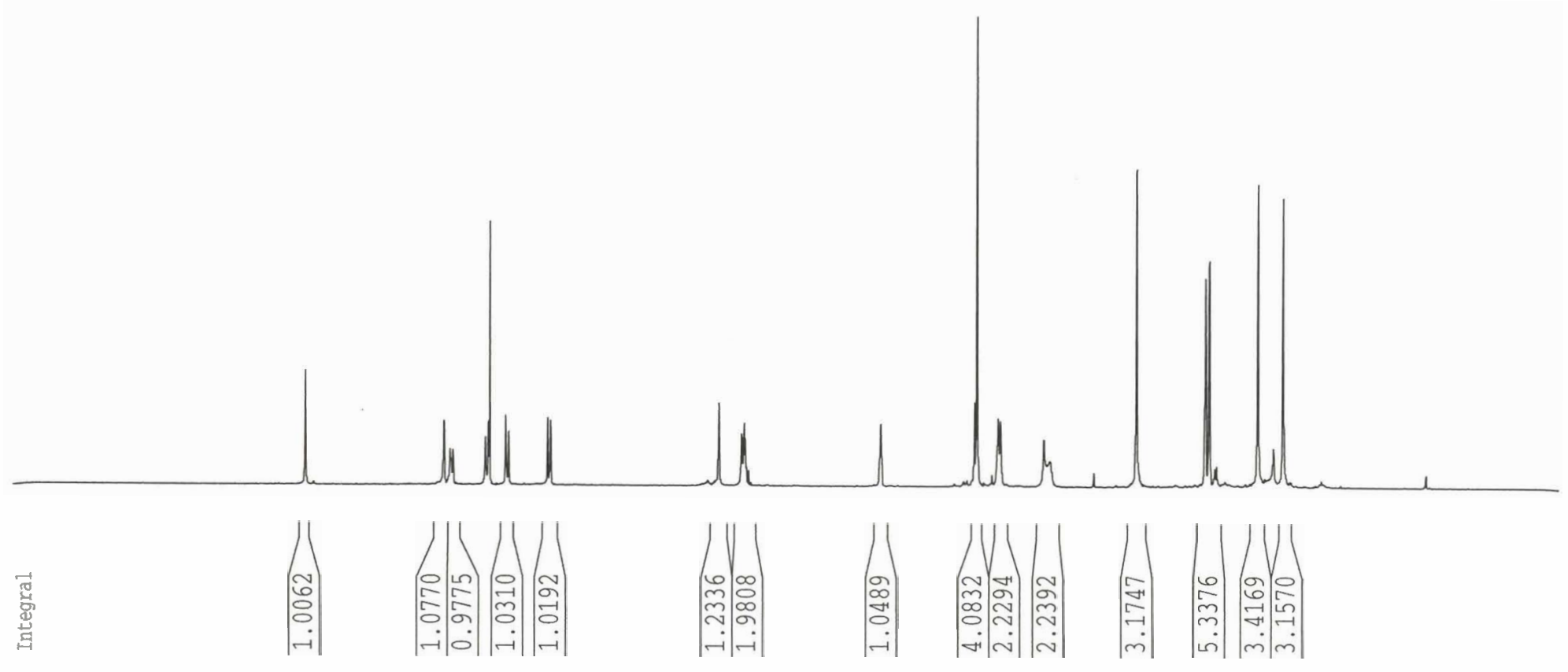

4
Current Data Parameters DHN 12-17

PROCNO

F2 - Acquisition Parameters

Date__ 20051217

Time 11.37

INSTRUM $\quad \operatorname{dr} \times 400$

PROBHD $5 \mathrm{~mm}$ QNP $1 \mathrm{H} / 13$

$\begin{array}{lr}\text { PULPROG } & 2 \mathrm{~g} 30 \\ \text { TD } & 32768\end{array}$

SOLVENT CDC13

DS

16

SWH $\quad 4789.272 \mathrm{~Hz}$

FIDRES $\quad 0.146157 \mathrm{~Hz}$

AQ $\quad 3.4210291 \mathrm{sec}$

$\begin{array}{lc}\text { RG } & 256 \\ \text { DW } & 104.400 \text { usec }\end{array}$

DE $\quad 5.50$ usec

TE $293.2 \mathrm{~K}$

D1 $\quad 1.00000000 \mathrm{sec}$

MCREST $\quad 0.00000000 \mathrm{sec}$

MCWRK $\quad 0.01500000 \mathrm{sec}$

$=======$ CHANNEL f1 $=======$

NUC1 $1 \mathrm{H}$

P1 9.35 usec

SF01 $400.1320007 \mathrm{MHz}$

F2 - Processing parameters

SI 32768

SF $\quad 400.1300047 \mathrm{MHz}$

WDW EM

SSB

LB

GB

1D NMR plot parameters

CX $\quad 20.00 \mathrm{~cm}$

CY $\quad 0.00 \mathrm{~cm}$

F1 $10.973 \mathrm{ppm}$

F2P $\quad-0.997 \mathrm{ppm}$

F2 $-398.73 \mathrm{~Hz}$

PPMCM $\quad 0.59846 \mathrm{ppm} / \mathrm{cm}$

HZCM $\quad 239.46359 \mathrm{~Hz} / \mathrm{cm}$ 


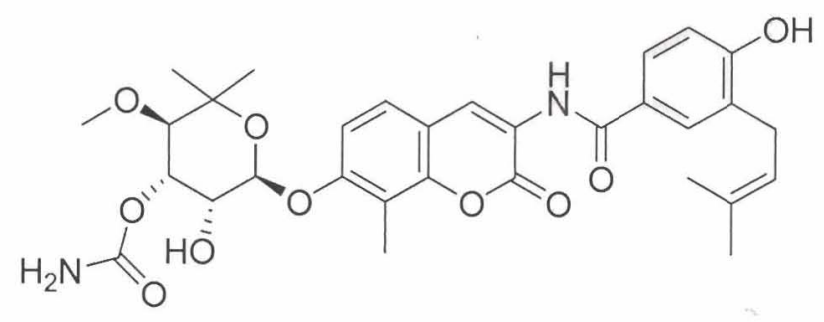

DHN1

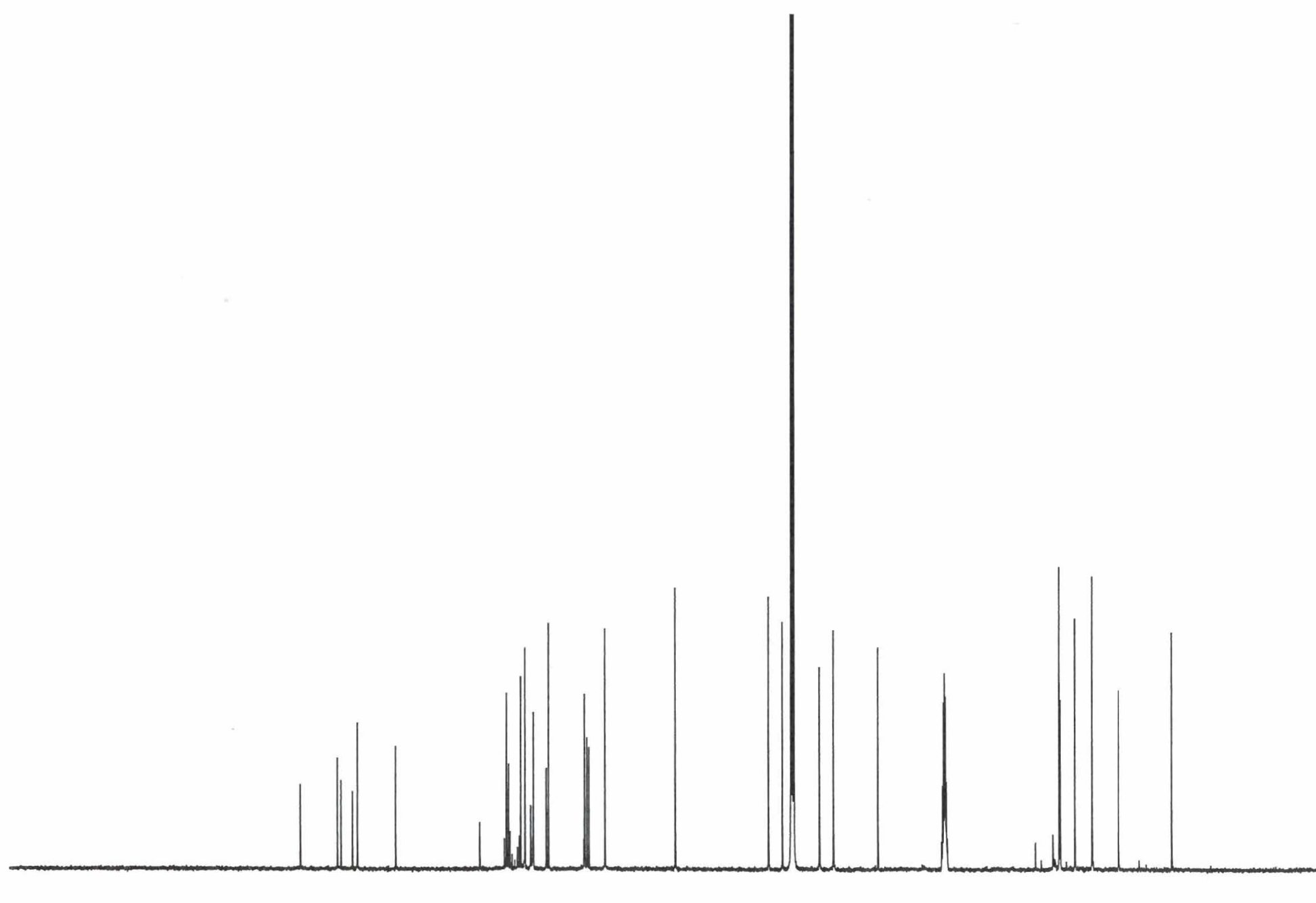

200

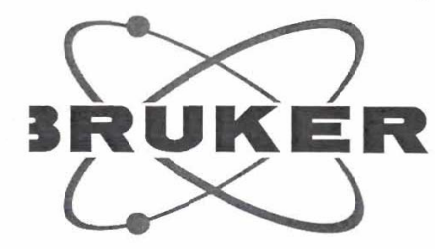

rrent Data Parameters ME

PNO

OCNO

2

F2 - Acquisition Parameters

20051216

$\begin{array}{lr}\text { Time } & 19.19\end{array}$

INSTRUM 5 spect

PROBHD $5 \mathrm{~mm}$ CPDUL $13 \mathrm{C}$
PULPROG

TD

SOLVENT

NS

SWH

FIDRES

$\mathrm{AQ}$
$\mathrm{RG}$

DW

$\mathrm{DE}$

TE

d11

DELTA

zgpg30

CDCI3

6144

$30030.029 \mathrm{~Hz}$

$0.458222 \mathrm{~Hz}$

$1.0912410 \mathrm{sec}$

13004

16.650 usec 6.00 usec $298.0 \mathrm{~K}$

$0.15000001 \mathrm{sec}$

$0.03000000 \mathrm{sec}$

$0.05000000 \mathrm{sec}$

TDO

$=======$ CHANNEL f 1

NUC1

PI

PI1 1

$13 \mathrm{C}$

9.95 usec

$3.80 \mathrm{~dB}$

CHANNEL $\mathrm{f} 2$ CPDPRG2 waltzi6

NUC2

PCPD2

PL2

PL12

PLI

80.00 usec

$2.20 \mathrm{~dB}$

$16.80 \mathrm{~dB}$

$10.00 \mathrm{~dB}$

$500.1320000 \mathrm{MHz}$

2 - Processing parameters

2 - Processing parameter
32768

$\begin{array}{lr}\text { SI } & 32768 \\ \text { SF } & 125.7577890 \mathrm{MHz}\end{array}$

DW

EM
0

$1.00 \mathrm{~Hz}$

0
1.40 


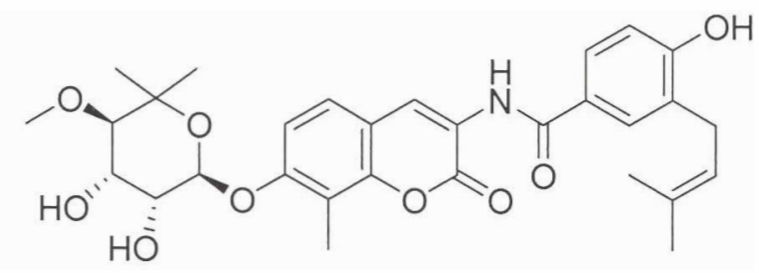

DHN2

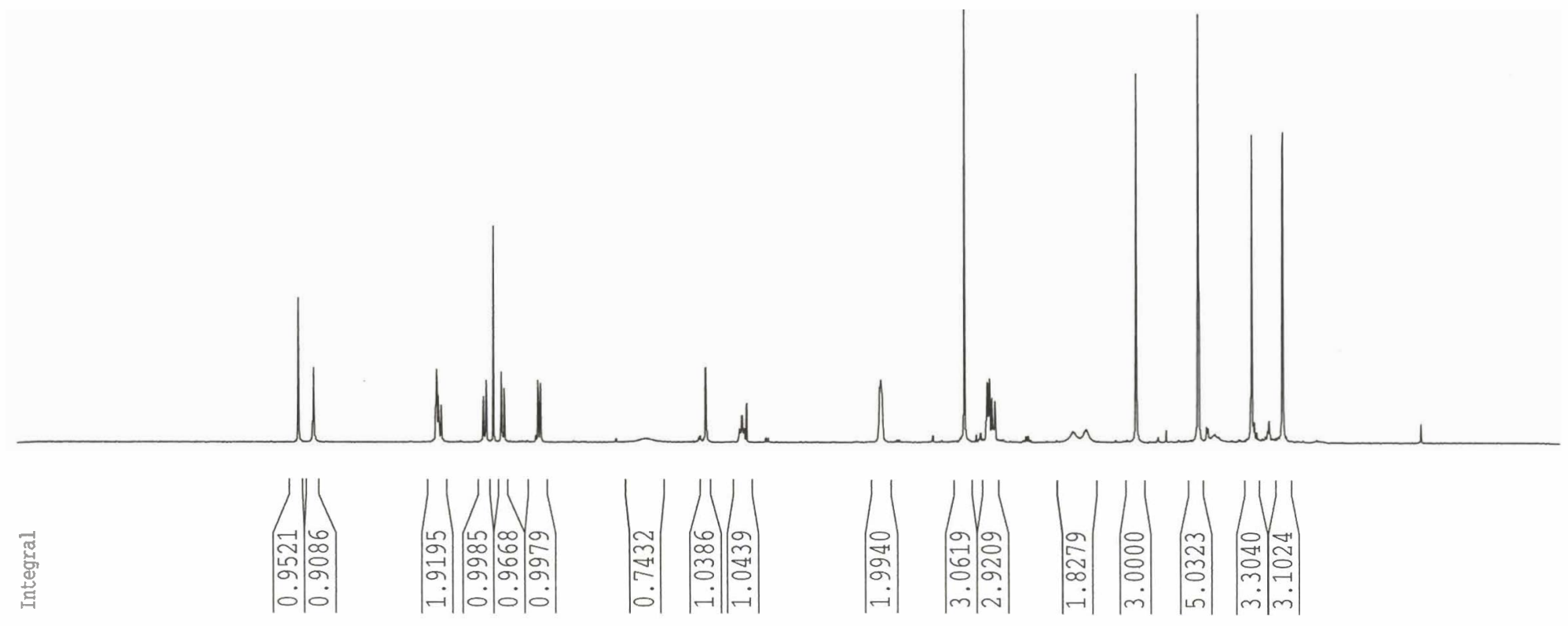

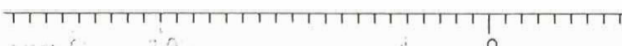
. 6010

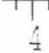

4
Current Data Parameters

NAME DHN diol 12-17

EXPNO

PROCNO

F2 - Acquisition Paramete S

Date_ 20051217

Time $\quad 11.50$

drx 400

PROBHD $5 \mathrm{~mm}$ QNP $1 \mathrm{H} / 13$

PULPROG $\quad \mathrm{zg} 30$

TD 32768

SOLVENT $\quad \mathrm{CDCl} 3$

NS

16

$\begin{array}{ll}\text { DS } & 2 \\ \text { SWH } & 4789.272 \mathrm{~Hz}\end{array}$

$\begin{array}{ll}\text { SWH } & 4789.272 \mathrm{~Hz} \\ \text { FIDRES } & 0.146157 \mathrm{~Hz}\end{array}$

$\mathrm{AQ} \quad 3.4210291 \mathrm{sec}$

RG 287.4

DW $\quad 104.400$ usec

DE $\quad 5.50$ usec

$293.2 \mathrm{~K}$

D1 $\quad 1.00000000 \mathrm{sec}$

MCREST $\quad 0.00000000 \mathrm{sec}$

MCWRK $\quad 0.01500000 \mathrm{sec}$

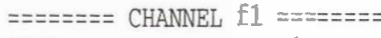

NUC1

PL1

9.35 usec
$-4.00 \mathrm{~dB}$

SF01 $\quad 400.1320007 \mathrm{MHz}$

F2 - Processing parameters

SI 32768

SF $\quad 400.1300091 \mathrm{MHz}$

WDW EM

$\begin{array}{lr}\text { WDW } & \text { EM } \\ \text { SSB } & 0\end{array}$

LB $\quad 0.30 \mathrm{~Hz}$

$\mathrm{PC}$

0
1.00

1D NMR plot parameters

CX $\quad 20.00 \mathrm{~cm}$

$\begin{array}{lr}\mathrm{CY} & 0.00 \mathrm{~cm}\end{array}$

F1P $\quad 10.962 \mathrm{ppm}$

F1 $4385.17 \mathrm{~Hz}$

F2P $\quad-1.007 \mathrm{ppm}$

F2 $-403.10 \mathrm{~Hz}$

PPMCM $\quad 0.59846 \mathrm{ppm} / \mathrm{cm}$

HZCM $\quad 239.46359 \mathrm{~Hz} / \mathrm{cm}$ 


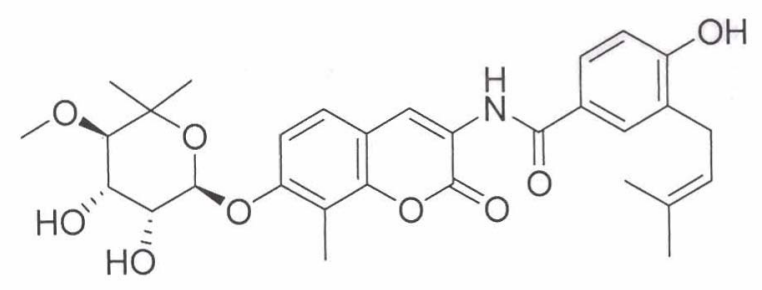

DHN2

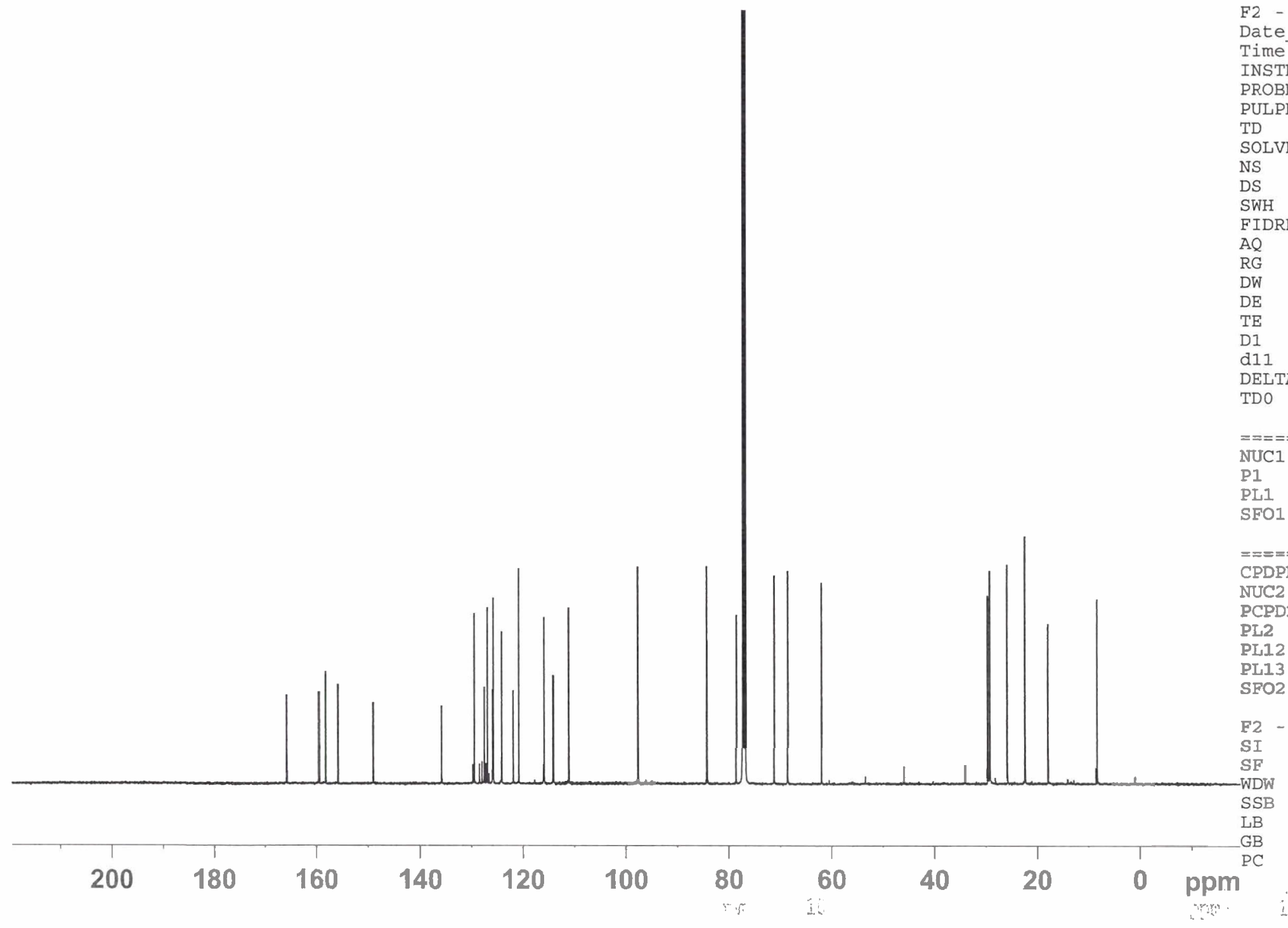

\section{B?}

Current Data Parameters NAME JAB_DHN_Diol EXPNO PROCNO

F2 - Acquisition Parameters Date_ 20051216

21.40

spect

zgpg30

OLVENT CDCl3

6144
NS

SWH $\quad 30030.029 \mathrm{~Hz}$

IDRES $0.458222 \mathrm{~Hz}$

$1.0912410 \mathrm{sec}$

13004

16.650 usec

6.00 usec

$298.0 \mathrm{~K}$

$0.15000001 \mathrm{sec}$

$0.03000000 \mathrm{sec}$

$\begin{array}{lr}\text { DELTA } & 0.05000000 \mathrm{sec} \\ \text { TDO } & 1\end{array}$

$======$ CHANNEL $f$

(1)

$13 \mathrm{C}$

9.95 usec

9.95 usec

$125.7703640 \mathrm{MHz}$

$=====\equiv$ CHANNEL f $2========$ waltz16

NUC2

PCPD2
PL2

L12

$\mathrm{PL} 13$
$\mathrm{SFO} 2$

$1 \mathrm{H}$

80.00 usec $2.20 \mathrm{~dB}$ $16.80 \mathrm{~dB}$

$5001320000 \mathrm{MHz}$

- Processing parameters

32768
$125 \quad 7577890$ EM SB 0 $1.00 \mathrm{~Hz}$

0
1.40 